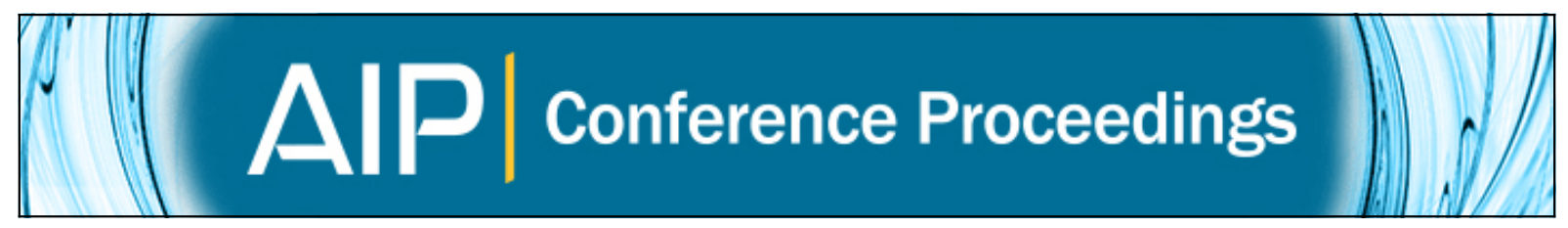

Statistical simulation of decaying and forced homogeneous isotropic turbulence

Fei Fei, Jing Fan, Chunxiao Xu, Yang Song, and Zhaohui Liu

Citation: AIP Conference Proceedings 1628, 1356 (2014); doi: 10.1063/1.4902749

View online: http://dx.doi.org/10.1063/1.4902749

View Table of Contents: http://scitation.aip.org/content/aip/proceeding/aipcp/1628?ver=pdfcov

Published by the AIP Publishing

Articles you may be interested in

The multiscale formulation of large eddy simulation: Decay of homogeneous isotropic turbulence

Phys. Fluids 13, 505 (2001); 10.1063/1.1332391

On the decay of homogeneous isotropic turbulence

Phys. Fluids 12, 1997 (2000); 10.1063/1.870447

Deterministic forcing of homogeneous, isotropic turbulence

Phys. Fluids 6, 1612 (1994); 10.1063/1.868274

The decay of homogeneous isotropic turbulence

Phys. Fluids A 4, 1492 (1992); 10.1063/1.858423

Decay of Passive Scalar Fluctuations in Isotropic Homogeneous Turbulence

Phys. Fluids 11, 671 (1968); 10.1063/1.1691967 


\title{
Statistical Simulation of Decaying and Forced Homogeneous Isotropic Turbulence
}

\author{
Fei Fei ${ }^{\mathrm{a}}$, Jing $\mathrm{Fan}^{\mathrm{b}}$, Chunxiao Xuc ${ }^{\mathrm{c}}$, Yang Song ${ }^{\mathrm{c}}$ and Zhaohui Liu ${ }^{\mathrm{a}}$ \\ ${ }^{a}$ State Key Laboratory of Coal Combustion, Huazhong University of Science and Technology, Wuhan 430074, China \\ ${ }^{b}$ State Key Laboratory of High Temperature Gas Dynamics, Institute of Mechanics, \\ Chinese Academy of Sciences, Beijing 100190, China \\ ${ }^{c}$ School of Aerospace, Tsinghua University, Beijing 100190, China
}

\begin{abstract}
The diffusive information preservation (D-IP) method, a molecule-based scheme, has been extended to simulate decaying and forced homogeneous isotropic turbulence, and validated by comparing with the corresponding DNS results. Furthermore, the Lagrangian statistics of relative dispersion in homogeneous isotropic turbulence calculated directly by D-IP show that molecular motion significantly affect the relative dispersion when the initial separations of molecular pairs are less than the Kolmogorov microscale.
\end{abstract}

Keywords: D-IP method, isotropic turbulence, relative dispersion.

PACS: 47.11.St, 47.27.Gs

\section{INTRODUCTION}

There is a great interest to extend molecular schemes such as the DSMC method [1] to multi-scale flows in order to understand and model the interaction between the microscopic and macroscopic transport processes [2], e.g. molecule dispersion [3] and chemical reactions [4] in turbulence, where the Knudsen number ( $\mathrm{Kn})$ is usually extremely small. However, the DSMC method becomes more and more inefficient when $K n$ decreases, due to its core assumption that decouples molecular motion and collisions each time step. Such assumption is valid only when the time step and cell size are smaller than the mean collision time and mean free path, respectively; otherwise the DSMC method will give rise to unphysical results $[5,6]$, which prevents its applications to gas flows with low Knudsen numbers.

Many efforts have been made to breakthrough these limitations in the DSMC method. Recently, Fei \& Fan [7] proposed the D-IP method that may be viewed as a combination of the Langevin approach [8-10] and the information preservation (IP) method [11-15]. The main idea of D-IP is to track the motion of a simulated molecule from the diffusive standpoint, and obtain the flow velocity and temperature through sampling and averaging the IP quantities. The D-IP method was validated in simulating benchmark problems, such as Couette flows, Poiseuille flows, Rayleigh flows and square cavity flows with $K n \sim 10^{-3}-10^{-4}$, and the results showed that the D-IP method was not only accurate but also efficient for the low Knudsen number flows.

This paper aims to simulate decaying and forced homogeneous isotropic turbulence by the D-IP method, and pays more attention to relative dispersion where molecular transport processes plays an important role $[3,16]$. Since the D-IP method is a molecular scheme, the Lagrangian properties [17, 18], such as the relative dispersion, may be obtained directly, which is convenient and helpful to examine the influence of the molecular transport processes on them.

This paper is organized as follows. In Sec. 2, the D-IP method is briefly introduced. In Sec. 3, D-IP is applied to simulate the decaying and forced homogeneous isotropic turbulences (HIT), respectively, and validated by comparing the results with those of the DNS method. The Lagrangian properties of relative dispersion with and without molecular diffusion are calculated and analyzed in Sections 4. Finally conclusions are given.

\section{DIFFUSIVE INFORMATION PRESERVATION METHOD}

The D-IP method is a molecule based numerical method for unsteady gas flows with low Kn number. And the simulated molecules (simulators) are exactly tracked by the Langevin equation, which is a good approximate equation for low $K n$ number gaseous molecular motion [7]. Furthermore, the IP velocity and temperature, which can

Proceedings of the 29th International Symposium on Rarefied Gas Dynamics

AIP Conf. Proc. 1628, 1356-1362 (2014); doi: 10.1063/1.4902749

(C) 2014 AIP Publishing LLC 978-0-7354-1265-1/\$30.00 
be considered as the collective velocity and temperature of a mass of molecules, are assigned to each simulator like in IP method. This collective information could be transported with the molecular motion, and changed according to the governing equation of momentum and energy in order to satisfy the correct transport process in physics. Then the flow field is calculated by sampling the IP velocity and temperature in the computational domain. In the following, some detailed descriptions of this numerical scheme corresponding to the simulations in turbulence will be given.

\section{Langevin Model}

In the D-IP method, simulators' velocity $c_{k, i}$ is divided into two parts: IP velocity $u_{k, i}$ and thermal velocity $c^{\prime}{ }_{k, i}$,

$$
c_{k, i}=u_{k, i}+c_{k, i}^{\prime}
$$

During a time step, if the IP velocity is constant, and the thermal velocity is determined by the Langevin equation, the displacement of the simulators can be written as,

$$
r_{k, i}^{t+\Delta t}=r_{k, i}^{t}+u_{k, i}^{t} \times \Delta t+\Delta r_{k, i}^{D}
$$

where the second and third terms on the right-hand side reflect the convective motion and thermal motion of a molecule, respectively. The thermal motion term can be sampled as,

$$
\begin{aligned}
& \Delta r_{k, i}^{D}=\left(-2 F \ln \Phi_{r}\right)^{1 / 2} \cos \alpha_{r}+c_{k, i}^{\prime}(t)[1-\exp (-\zeta \Delta t)] / \zeta, \\
& c_{k, i}^{\prime}(t+\Delta t)=\left[-\frac{2\left(F G-H^{2}\right)}{F} \ln \Phi_{r}\right]^{1 / 2} \cos \alpha_{r}+\frac{H \times R_{i}}{F}+c_{k, i}^{\prime}(t) \exp (-\zeta \Delta t),
\end{aligned}
$$

which are the same as Equations (5) and (6) in ref. [7], where $\Phi_{r}$ and $\alpha_{r}$ are random numbers uniformly distributed in $[0,1]$ and $[0,2 \pi]$, respectively, and other parameters can also be found at section 1 in [7].

\section{Governing Equation of IP Velocity}

Similarly to the IP method, the IP velocity and temperature remain constant during the molecular motion, and should be updated according to their governing equations to maintain the correct momentum and energy transport. In the simulation of HIT, the Mach number is less than 0.1, and the compressive effect is small. Therefore, the energy equation is not considered, we only rewrite the governing equation for IP velocity here from [7],

$$
\frac{\partial}{\partial t}\left(n m u_{i}\right)+\underbrace{\frac{\partial}{\partial x_{j}}\left(n m u_{i} u_{j}\right)}_{\text {collective motion }}+\underbrace{\frac{\partial}{\partial x_{j}}\left(n m u_{i} c_{j}^{\prime}\right)}_{\text {thermal motion }}=\underbrace{-\frac{\partial p}{\partial x_{i}}+\underbrace{\Delta\left[m c_{i}\right]}_{\text {collsion }}+\rho a_{i}^{F}\left(x_{k}, t\right)}_{\begin{array}{c}
\text { viscocitymodified } \\
\text { pressure modified }
\end{array}} .
$$

The left-hand and right-hand side of Eq. (5) corresponds to the molecular motion and the IP velocity updated model for each simulator, respectively.

When the $K n$ numbers is small, the temporal and spatial scales are much larger than those of molecular motion, therefore the local equilibrium condition prevails. And the pressure term as the third term on the right-hand side of Eq. (5) is consistent with expression from local equilibrium approach in [13].

Furthermore, the viscosity modified terms as the first two terms on the right-hand side of Eq. (5) are divided into two parts due to incompressible and compressible effects, respectively. They are satisfied with the momentum transport law in the continuum region when the Kn number becomes small. The modified coefficient, $\psi_{\eta}=\mu /\left(\rho D^{*}\right)$, 
where $D^{*}=D\left[1-\left(1-e^{-\zeta \Delta t}\right) / \zeta \Delta t\right], \mu$ and $D$ are viscosity and diffusion coefficients, respectively. As the time step $\Delta t \rightarrow \infty, \psi_{\eta}=\mu /(\rho D)$, which is the Schmidt number $(S c)$, and it implies that in the D-IP method the momentum transport is determined by diffusion process.

The fourth term on the right-hand side of Eq. (5) is the collision term. Since the time step of D-IP is usually much larger than mean collision time, it is reasonable to assume that the IP velocity at $t+\Delta t$ is equal to the mean values of its surrounding simulators, i.e.

$$
u_{k, i}^{t+\Delta t}=\frac{1}{N} \sum_{j=1}^{N} u_{j, i}^{t+\Delta t}
$$

where $N$ is the number of simulators in a neighboring region around simulator $\mathrm{k}$, the regional size can be chosen according to the spatial scales and computational accuracy of flows.

The last term on the right-hand side of Eq. (5) relates to the forcing acceleration, which are zero for the decaying HIT and non-zero for the forced HIT. The stochastic forcing scheme proposed by Eswaran and Pope [19] has been used here. In their scheme, the random forcing acceleration $a_{i}^{F}(\mathbf{k}, t)$ is added to the low wavenumbers of velocity field in the wave number space $\mathbf{k}$ in order to keep the HIT. However, the D-IP method is solved in the physical space through simulators, the forcing acceleration should be transformed to the physical space by FFT (Fast Fourier Transform) firstly at each time step,

$$
a_{i}^{F}(\mathbf{k}, t) \stackrel{F F T}{\longrightarrow} a_{i}^{F}(\mathbf{x}, t)
$$

Then by interpolating it into the location of simulators, the IP velocity acceleration for each simulator, $a_{i}^{F}\left(x_{k}, t\right)$, can be obtained.

\section{Numerical Implementation}

Similar to the conventional IP method, there are six steps to implement the D-IP method.

1. Initialization. Each simulator is initially given a position $r_{k, i}(\mathrm{k}=1,2, \ldots, \mathrm{N})$, a thermal velocity $c_{k, i}^{\prime}$, an IP velocity $u_{k, i}$ according to initial gas flow field. In the simulation of HIT, the initial field is obtained from pseudo-spectral method, whose velocity field is stored in the wavenumber space. In order to initialize the IP velocity quickly, simulators are placed in uniform nodes initially, i.e. $8^{3}$ nodes in each computational cell in this work, and then the FFT method can be used to transform the velocity from the wavenumber space to the physical space. The initial IP velocity of each simulator equals to the flow velocity at related nodes.

2. Molecular motion. After a time step, simulator $\mathrm{k}$ acquires a new position and thermal velocity according to the Langevin model.

3. Update IP velocity. After a time step, the IP velocity of simulator $\mathrm{k}$ is updated according to the governing equation (5). Especially in the simulation of forced HIT, the forcing acceleration should be calculated in every time step.

4. IP velocity changed due to collisions.

5. Interact with boundaries. In HIT simulation, the periodic boundary condition is used.

6. Sample and average. The flow velocity is obtained by sampling the IP velocity of simulators in each cell, $U_{i}=\bar{u}_{i}$. And the flow number density is calculated using the mass conservation equation with finite volume scheme,

$$
\frac{\partial(n m)}{\partial t}+\frac{\partial\left(n m U_{j}\right)}{\partial x_{j}}=0 .
$$

The basic characters in HIT are turbulent kinetic energy $E_{T}$ and energy dissipation $\varepsilon$. In D-IP method, they can be directly obtained from their definition in physics, 


$$
\begin{gathered}
E_{T}=\frac{1}{2}\left\langle u^{2}\right\rangle=\frac{1}{N} \sum_{k=1}^{N} \frac{1}{2} u_{k}^{2} \\
\varepsilon=\frac{d E_{T}}{d t}=\frac{E_{T}\left(t_{0}+\Delta t\right)-E_{T}\left(t_{0}\right)}{\Delta t}
\end{gathered}
$$

Then the other turbulent characters used in this paper can be calculated from $E_{T}$ and $\varepsilon$, such as Taylor microscale $\lambda_{T}=\sqrt{5 v\left\langle U^{2}\right\rangle / \varepsilon}$, Reynolds number $\operatorname{Re}_{\lambda}=\left[\lambda_{T} \sqrt{\left\langle U^{2}\right\rangle / 3}\right] / v$, Kolmogorov microscale $\eta=\left(v^{3} / \varepsilon\right)^{1 / 4}$, and Kolmogorov time-scale $t_{\eta}=(v / \varepsilon)^{1 / 2}$.

\section{DECAYING AND FORCED HOMOGENEOUS ISOTROPIC TURBULENCE}

In present work on turbulent simulation, the flow medium is argon gas in standard condition ( 1 atm and $273 \mathrm{~K}$ ). Two kinds of periodic cubic computational domains with $\left(3.25 \times 10^{4} \mathrm{~m}\right)^{3}$ for $64^{3}$ grids and $\left(1.625 \times 10^{-4} \mathrm{~m}\right)^{3}$ for $32^{3}$ grids are used, and the cell size is $78 \lambda$, where $\lambda$ is mean free path; furthermore, 512 simulated molecules are initially placed in each grid. The time step of all cases is $50 \tau_{c}$, where $\tau_{c}$ is mean collision time. Since the Mach number of simulated turbulence is small, energy equation is not considered here. Therefore, temperatures of the decaying and forced HIT are both assumed to be constant at $273 \mathrm{~K}$.

\section{Decaying Homogeneous Isotropic Turbulence}

When simulating the decaying HIT, a fully developed HIT is arranged as the initial flow field. The decaying process of this HIT is calculated by D-IP method and pseudo-spectral method, respectively. The evolution of the turbulent kinetic energy and energy dissipation are shown in Figure 1, as well as the Kolmogorov-scaled dissipation spectra at two marked times 1 and 2. Furthermore, related vorticity at marked time 1 is displayed in Figures 2. In these figures, $E_{T 0}, \varepsilon_{0}$, and $t_{\eta}$ are turbulent kinetic energy, energy dissipation and Kolmogorov time-scale at the initial time, respectively. The results of the D-IP method are almost identical with that of the pseudo-spectral method with the same grid, which demonstrates the validity of the D-IP method for the turbulence simulation.

\section{Forced Homogeneous Isotropic Turbulence}

Forced HIT, the turbulent flow persisted by adding forcing from outside, is more useful than decaying HIT when calculating and analyzing the statistic properties of turbulence. In the simulation of forced HIT, the stochastic forcing scheme given by Eswaran and Pope is used. To verify the D-IP method, two cases such as f28 and f33 in ref. [19], presented in Table 1, have been calculated. Evolution of the turbulent kinetic energy, energy dissipation, and the Reynolds number based on Taylor microscale $\mathrm{Re}_{\lambda}$ have been demonstrated in Fig. 3, as well as related Kolmogorov-scaled dissipation spectra. Where $E_{T 0}, \varepsilon_{0}$, and $\operatorname{Re}_{\lambda 0}$ are the simulated values in average in [19], respectively. The results of D-IP method agree well with the reference, which also validate the D-IP method.

It is important to notice that the cell size and the time step in both decaying and forced HIT simulations are as large as $78 \lambda$ and $50 \tau_{c}$, where the length of computational domain is about $5000 \lambda$, and the flow evolution time is 30 eddy-turnover time, about $6.9 \times 10^{5} \tau_{c}$. To simulate these cases, the computational cost will be very huge for traditional molecule-based method, such as DSMC. And D-IP method should cost about one week by using 128 CPU cores, more than the pseudo-spectral method, but it is the first time to simulate the turbulence with a moleculebased method. Therefore, the D-IP method enables us to investigate the turbulence at molecular level. 


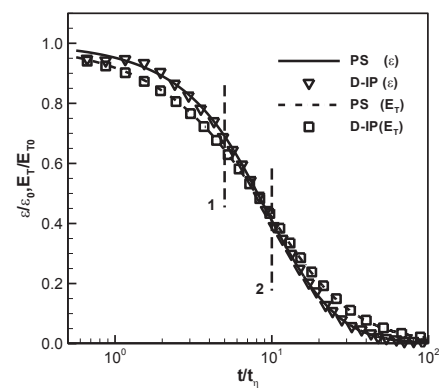

(a)

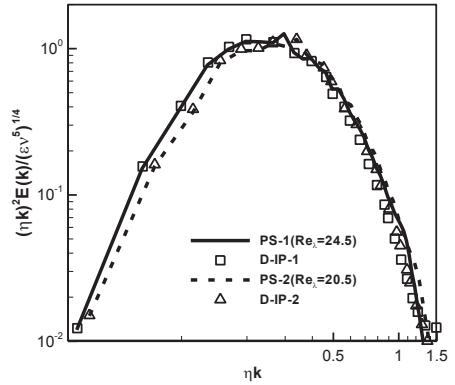

(b)

FIGURE 1. (a) Evolution of the turbulent kinetic energy $E_{T}$ and energy dissipation $\varepsilon$; and (b) the Kolmogorov-scaled dissipation spectra at time 1 and 2 simulated by the D-IP/pseudo-spectral method.
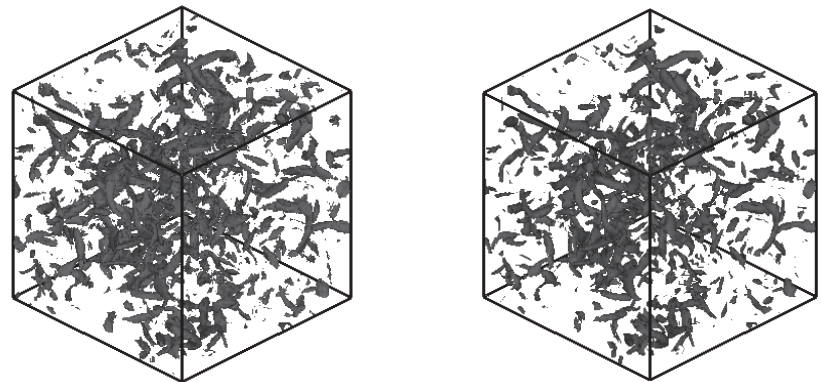

FIGURE 2. Vorticity contour $\left(8 \times 10^{6} s^{-1}\right)$ of D-IP (left) and pseudo-spectral (right) method at time 1 .
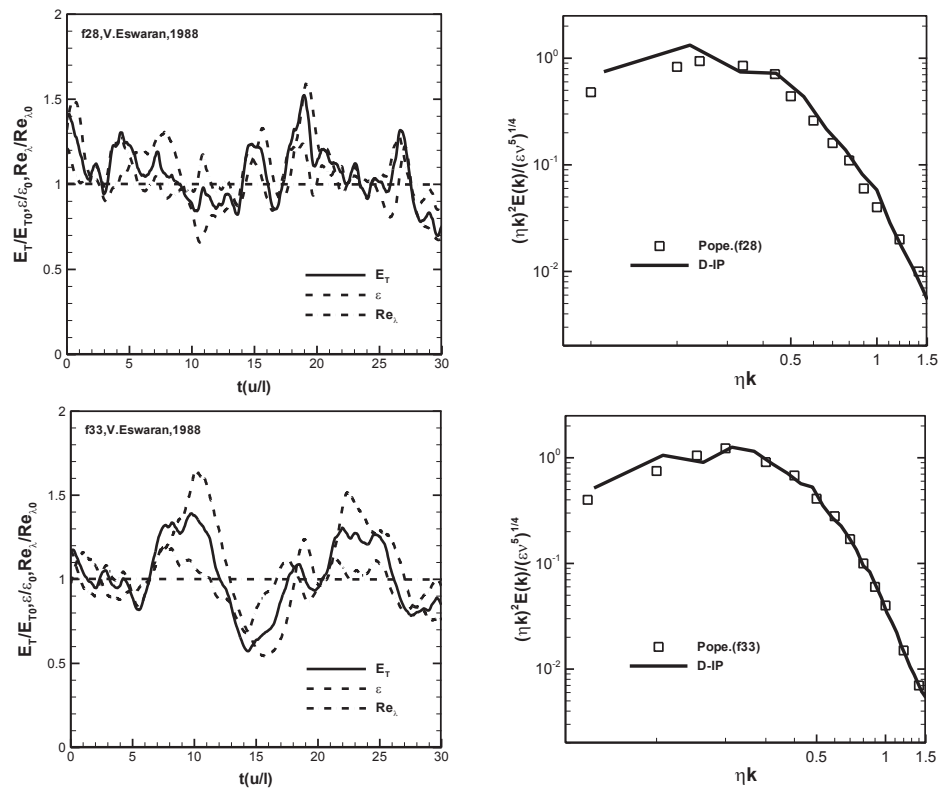

FIGURE 3. Evolution of the turbulent kinetic energy $E_{T}$, energy dissipation $\varepsilon$ and $\operatorname{Re}_{\lambda}$ versus time normalized by the eddyturnover time (left); and the Kolmogorov-scaled dissipation spectra (right) of case f28 (up) and f33 (down).

\section{RELATIVE DISPERSION IN HOMOGENEOUS ISOTROPIC TURBULENCE}

Turbulent relative dispersion is the spreading of a pair or a cloud of particles relative to their center of mass, which is important for understanding the mixing and concentration fluctuation of scalars in turbulence. When the initial source size is compared with the Kolmogorov microscale, effect of the molecular motion should not be ignored $[3,16]$. In present paper, D-IP method is used to calculate the Lagrangian statistics of turbulence in order to investigate how the molecular motion influencing relative dispersion in forced HIT. The simulated domain is $64^{3}$ 
grids, and other computational parameters are the same as those in Sect. 3. The forcing parameters in dimensionless form, which are identical with [19], are given in the third line in Table 1.

Sawford and coworkers [20] have studied the Reynolds number dependence of relative dispersion in HIT by considering the marked fluid particle (without molecular motion). Using their statistical method, the differential displacement covariance for single particle is defined as,

$$
F_{i j}\left(\mathrm{t}_{1}, \mathrm{t}_{2}\right)=\left\langle\hat{x}_{i}\left(\mathrm{t}_{1}\right) \hat{x}_{j}\left(\mathrm{t}_{2}\right)\right\rangle \text {, }
$$

where $\hat{x}=x-u_{0} t-x_{0}$, and $u_{0}=u(0), x_{0}=x(0)$; i.e., an inertial reference frame moving with the particle initial velocity is considered. Similar to the single particle, the relative differential displacement covariance for pair of particles is defined as,

$$
F_{i j}^{(r)}\left(\mathrm{t}_{1}, \mathrm{t}_{2}\right)=\left\langle\hat{x}_{i}^{(r)}\left(\mathrm{t}_{1}\right) \hat{x}_{j}^{(r)}\left(\mathrm{t}_{2}\right)\right\rangle
$$

where $\hat{x}^{(r)}=\hat{x}^{(1)}-\hat{x}^{(2)}$, and the superscript notations (1) and (2) are represented each particle in pair. The relative differential displacement covariance displays structures of displacement between relative pairs.

In order to investigate the effect of molecular motion in relative dispersion in HIT, the relative differential displacement covariance of gas molecules instead of the marked fluid particles should be considered, which can statistic from the D-IP method directly. Molecule pairs with three initial separations, such as $0.25 \eta, \eta$ and $4 \eta$, are calculated, and their results are shown in Fig. 4 with solid lines. Additionally, the relative dispersion of marked fluid particles is also calculated as reference in the same situations (dot-dash lines in Fig. 4). The marked fluid particles without influence of the turbulent flow are added to the flow field at initial time, and their velocities are obtained by averaging the IP velocity of simulators around.

In Fig. 4, compared with the marked fluid particles' results, we can find that the effect of molecular motion is more significant in relative dispersion than in the case of single molecule. For single particle dispersion, influence of molecular motion is only obvious at early times (less than tens of Kolmogorov time-scale) in turbulence, but it can maintain for hundreds of Kolmogorov time-scale for pair's relative dispersion. Furthermore, as the initial separation

TABLE 1. Dimensionless parameters for forcing HIT

\begin{tabular}{ccccccc}
\hline Case & $\mathrm{N}_{\text {cell }}$ & $\mathrm{K}_{\mathrm{F}}$ & $\varepsilon^{*}$ & $T_{L}{ }^{*}$ & $\mathrm{Re}^{*}$ & $\mathrm{Re}_{\lambda}$ \\
\hline $\mathrm{F} 28$ & 32 & $\sqrt{2}$ & 0.0033 & 0.201 & 5.96 & 28.2 \\
$\mathrm{~F} 33$ & 64 & $\sqrt{2}$ & 0.0760 & 0.360 & 16.64 & 53.9 \\
Relative dispersion & 64 & $2 \sqrt{2}$ & 0.0145 & 0.200 & 9.75 & 38 \\
\hline
\end{tabular}

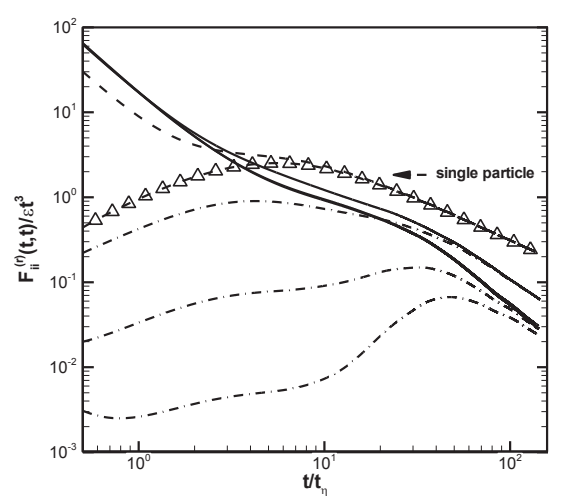

(a)

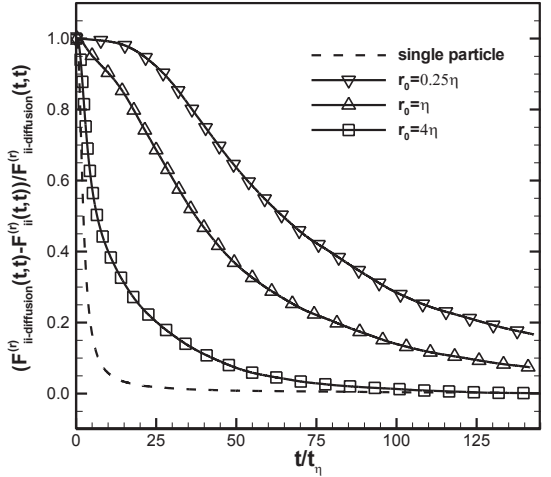

(b)

FIGURE 4. (a) Influence of molecular motion to the mean-square relative dispersion. The dash line and symbols are for single particle, and the solid and dot-dash lines are for pair of particles, with and without molecular motion, respectively. From bottom to top, $r_{0} / \eta=0.25,1$, and 4 , are both for the solid and dot-dash lines. (b) The relative importance of molecular motion to relative dispersion in HIT for different initial pair separations. 
decreasing, influence of molecular motion becomes more important, as shown in the right hand of Fig. 4. These results imply that the relative dispersion is more influenced by the small structure in turbulence, where the effect of molecular motion is significant. It is also consistent with the consequences in [3] and [16]. Since molecular motion is isotropic, it is independent of pair's separate distance as well as the turbulent velocity structure. Therefore, it is interesting to note, when the initial separation is $0.25 \eta$ and $\eta$ less than one Kolmogorov microscale, the effect of molecular motion is dominated, and it makes pairs' relative dispersions almost the same even with different initial separation, as in the left hand of Fig. 4.

Briefly speaking, as the sources' character scale decreasing below the Kolmogorov microscale, effect of molecular motion becomes more important, especially for relative motion of molecules. The situation will be more complicated in turbulent combustion. Since chemical reaction is always happened in small scale, the interaction between molecular motion and turbulent fluctuation will be certainly significant [4]. It is looking forward to applying the D-IP method to investigate that in future work.

\section{CONCLUSION}

The D-IP method has been used to simulate the decaying and forced homogeneous isotropic turbulence, with the Reynolds number based on the Taylor microscale between 20 50. The comparison between the D-IP and DNS results shows that they are in good agreement. One of the advantages for D-IP method in simulating turbulent flows is to obtain molecular Lagrangian statistics directly. Effect of molecular motion on the relative dispersion in isotropic turbulence has been investigated using D-IP method. The results imply that the effect is important, especially when the initial separations of molecular pairs are less than Kolmogorov microscale.

\section{ACKNOWLEDGMENTS}

This work was supported by China Postdoctoral Science Foundation and National Natural Science Foundation of China under Grant Nos. 51276076, 51306065, \& 51390494.

\section{REFERENCES}

1. G.A. Bird, Molecular Gas Dynamics and Direct Simulation of Gas Flows, Clarendon Press, Oxford, 1994.

2. J. Fan. Advances in Mechanics, 43, 185-201 (2013) .

3. B. L. Sawford, J. C. R. Hunt, J. Fluid Mech. 165, 373-400 (1986).

4. R. Hilbert, F. Tap, H. El-Rabii, D. Thevenin, Prog. Energy Combust. Sci. 30, 61-117 (2004).

5. F.J. Alexander, A.L. Garcia, B.J. Alder, Phys. Fluids 10, 1540-1542 (1998).

6. N.G. Hadjiconstantinou, Phys. Fluids 12, 2634-2638 (2000).

7. F. Fei, J. Fan, J. Comput. Phys. 243, 179-193 (2013).

8. P. Jenny, M. Torrilhon, S. Heinz, J. Comput. Phys. 229, 1077-1098 (2010).

9. M.H. Gorji, M. Torrilhon, P. Jenny, J. Fluid Mech. 680, 574-601 (2011).

10. M.H. Gorji, P. Jenny, J. Comput. Phys. 262, 325-343 (2014).

11. J. Fan, C. Shen, "Statistical simulation of low-speed unidirectional flows in transition regime" in Rarefied Gas Dynamics 21 , edited by R. Brun, Cepadus-Editions, Toulouse, 1999, pp. 245-252.

12. J. Fan, C. Shen, J. Comput. Phys. 167, 393-412 (2001).

13. Q. Sun, I.D. Boyd, "Theoretical development of the information preservation method for strongly nonequilibrium gas flows", AIAA 2005-4828 (2005).

14. N.D. Masters, W. Ye, J. Comput. Phys. 226, 2044-2062 (2007).

15. J. Zhang, J. Fan, J. Jiang, J. Comput. Phys. 230, 7250-7265 (2011).

16. M. S. Borgas, B. L. Sawford, J. Fluid Mech. 324, 25-54 (1996).

17. P. K. Yeung, Ann. Rev. Fluid Mech. 34, 115-142 (2002).

18. F Toschi, E. Bodenschatz, Ann. Rev. Fluid Mech. 41, 375-404 (2009).

19. V. Eswaran, S. B. Pope, Comput. \& Fluids 16, 257-278 (1988).

20. B. L. Sawford, P. K. Yeung, J. F. Hackl, Phys. Fluids 20, 065111 (2008). 